\title{
Explanation and Intergroup Emotion: Social Explanations as a Foundation of Prejudice-Related Compunction
}

\author{
Michael J. Gill and Michael R. Andreychik \\ Lehigh University
}

\begin{abstract}
Two studies examined whether social explanations — causal frameworks used to make sense of a group's status and behavior-are associated with prejudice-related compunction. In Study 1, based on Devine, Monteith, Zuwerink, \& Elliott, (1991), participants who endorsed external explanations (e.g. low socioeconomic status of Blacks stems from historical maltreatment) showed a particularly strong tendency to experience compunction in response to prejudicerelated discrepancies. Study 2 involved a novel paradigm. Participants were induced to admit that they would discriminate against Black males. Conceptually replicating Study 1 , endorsement of external explanations was positively associated with compunction in response to this imagined discrimination. Across both studies, there was also evidence that the effects of external explanations are not explicable in terms of internal motivation to avoid prejudice, global prejudice, or global positive evaluation of African Americans. Discussion centers on the importance of explanations in shaping intergroup emotions and how the concept of explanation links the intergroup emotion literature to other emotion literatures.
\end{abstract}

KEYWORDS prejudice-related compunction, social explanations

Social psychologists have long sought to understand the nature of prejudice and the keys to its reduction (see Oskamp, 2000). Recently, researchers have focused their attention on Allport's (1954) notion of prejudice with compunction as integral to prejudice reduction efforts. The idea is that if people experience compunction-negative, self-directed emotions (e.g. guilt, anger at self)—following a prejudicial response, this initiates a self-regulatory cycle that fosters prejudice reduction in both the short-and long-term (e.g. Devine et al., 1991; Monteith, 1993). Following from this, some researchers have attempted to pinpoint belief systems that predispose people to the experience of prejudicerelated compunction (e.g. Plant \& Devine, 1998). The present work continues in this tradition by examining whether social explanations - the causal frameworks people employ to understand group status and action-are associated with the

\footnotetext{
$\overline{\text { Author's note }}$

Address correspondence to Michael J. Gill, Department of Psychology, Lehigh University, 17 Memorial Drive East, Bethlehem, PA, 18015, USA [email: m.gill@lehigh.edu]
} 
degree of compunction experienced following a prejudicial response.

\section{Prejudice with or without compunction}

Devine (1989) made the seminal argument that prejudicial responses can be spontaneously triggered upon encountering members of stereotyped groups. More surprisingly, she argued, this can occur even among people who desire to be unbiased. Those who desire to be unbiased must engage in effortful mental activity to inhibit biased responses and replace them with unbiased responses. This conceptual framework-involving ideas of spontaneous bias and effortful debiasing - has become highly influential (e.g. Bargh, 1999; Fiske, Lin, \& Neuberg, 1999; but see Fazio, Jackson, Dunton, \& Williams, 1995; Lepore \& Brown, 1997; Moskowitz, Gollwitzer, Wasel, \& Schaal, 1999, for evidence that unbiased responses can be spontaneous). Importantly, this framework implies that people are not simply prejudiced or unprejudiced. Rather, many people are ambivalent (Katz \& Hass, 1988) and characterized by prejudice-related discrepancies: prejudicial responses that exist alongside a sense that those responses are undesirable.

Devine et al. (1991) explored people's emotional reactions to awareness of their prejudicerelated discrepancies. They found that many participants reported that they would respond to a member of a stereotyped group (African Americans in Study 1; homosexual men in Study 2) in more prejudiced ways than they believed they should. For example, many participants indicated that they would be uncomfortable if a Black person or gay male sat next to them on the bus, even though they believed that they should not experience such discomfort. Critically, participants had divergent emotional reactions when they were made aware of these prejudicerelated discrepancies: those low in prejudice toward the group experienced compunction, whereas those high in prejudice experienced only general discomfort.

Monteith and her colleagues (1993; Monteith, Ashburn-Nardo, Voils, Czopp, 2002) went further to explore the notion that prejudice-related compunction motivates self-regulation of prejudice. For example, Monteith (1993)found that among those who experienced prejudicerelated compunction there were associated tendencies toward self-focus, thoughts concerning one's discrepancies, attention to discrepancy-relevant information, and, critically, long-term inhibition of prejudiced responses (e.g. subsequently heard gay-related jokes were evaluated less favorably). Furthermore, Monteith et al. (2002) presented evidence suggesting that-among those low in prejudice toward African Americans-the perception of having been biased gave rise to compunction, behavioral inhibition, and reflection (which are theoretically necessary for an effortful process of self-regulation), and more positive subsequent responses to African Americans. Taken together, these findings suggest that the experience of compunction can trigger a self-regulatory cycle directed toward prejudice reduction.

\section{The psychological bases of prejudice- related compunction}

The literature thus reveals that people sometimes show biases that they simultaneously consider undesirable. Moreover, when such prejudice-related discrepancies occur, some individuals experience compunction. Finally, this compunction affects whether individuals will engage in self-regulatory processes tailored toward the reduction of their biases. It is also clear that, for some individuals, prejudicial responses do not foster compunction and thus are unlikely to energize debiasing efforts. If the experience of prejudice-related compunction is important for prejudice reduction, it is crucial to understand its underlying foundations. That is, why do some individuals experience prejudicerelated compunction whereas others do not?

Plant and Devine (1998) provided an illuminating answer to this question: they examined the source of people's motivation to respond in unbiased ways and suggested that this is a critical factor affecting emotional reactions to prejudice-related discrepancies. Specifically, they posited that individuals who are high in internal motivation to avoid bias will experience 
compunction as a result of such discrepancies. Such individuals have anti-bias value systems that are central to their self concept (e.g. 'being a good person requires me to shun prejudice'). Violating this deeply held value system results in feelings of compunction, as does the violation of any internalized 'ought' standard (Higgins, 1987). In contrast, individuals high in external motivation to avoid bias will not experience compunction. For these individuals, eliminating bias is simply an attempt to conform to perceived societal standards (e.g. 'people might not like me if they see that I am biased'), and has no deep basis in the individual's value system. Hence, violating these standards does not foster compunction. Plant and Devine (1998) reported data supporting this framework: those high in internal motivation are especially likely to experience compunction when made aware of their prejudice-related discrepancies.

\section{Social explanations as a foundation of compunction}

The intended contribution of the present article is to offer-and provide empirical support for-a different conceptual approach to understanding prejudice-related compunction. Our analysis is based on the concept of social explanations, which are the causal frameworks people rely on to make sense of societal patterns of group status and group action. Explanation is a fundamental and pervasive cognitive activity aiding the development of knowledge about the world that can provide a basis for action (e.g. Gopnik, 2000; Heider, 1958). We take the view of scholars such as Heider (1958), Gilbert (1998), Malle (2004), and Weiner (2006) that explanation lies at the heart of social cognition. Our focus, however, is at the intergroup level, rather than the interpersonal level generally favored by these other scholars.

We start with the idea that people are motivated to explain salient facets of intergroup status and action (e.g. Why do African Americans predominate in so many decaying neighborhoods?). Stereotypes and prejudice are one possible consequence of this explanatory drive: these arise when people generate internal explanations-explanations that focus on features ostensibly inherent in the group (e.g. African Americans are irresponsible) - to explain intergroup patterns (see Allport, 1954; Hoffman \& Hurst, 1990; Jost \& Banaji, 1994; Sidanius \& Pratto, 1999; Yzerbyt \& Rocher, 2002). Despite receiving the bulk of attention in the intergroup attitudes literature, it is clear that internal explanations are not the only framework of explanation available to perceivers (see Gilbert, 1998; Heider, 1958; Malle, 2004; Weiner, 1986). Indeed, researchers have found that educational experiences, particularly university-level study in the social sciences, tend to foster external explanations - explanations that focus on factors 'outside of' a group (e.g. societal and economic forces) - as a determinant of intergroup patterns (Guimond, Begin, \& Palmer, 1989; Lopez, Gurin, \& Nagda, 1998). ${ }^{1}$ Although existing theory and research point strongly to the existence of internal and external explanatory frameworks within the realm of intergroup cognition, little evidence exists pertaining to the role of these frameworks in shaping intergroup emotion, cognition, and behavior more generally. ${ }^{2}$

Our lab has recently begun to explore these relations. For example, Gill and Andreychik (2006) had participants provide open-ended social explanations regarding the low socioeconomic status (SES) of African Americans (Study 1), the violence of terrorists (Study 2), or the kindness and caretaking tendencies of women (Study 3). Participants showed a clear tendency to reason in terms of internal and external causes. Moreover, across these diverse target groups, external explanations were associated with more positive orientations toward a group than were internal explanations. Specifically, those who generated external explanations were more supportive of diversity policies that could benefit African Americans (Study 1), more resistant to using violence to combat terrorism (Study 2), and had lower levels of hostile sexism (Study 3; see Glick \& Fiske, 1996). Furthermore, Study 4 provided evidence consistent with a causal role of social explanations. In that study, exposure to documentary footage highlighting external forces that have hindered African Americans reduced the internality of people's explanations 
and this shift in explanations mediated a shift toward more favorable social policy stances.

We expect that social explanations are relevant to the experience of prejudice-related compunction. Why? Our reasoning is rooted in the concept of the justice motive (Lerner, 1980; Ross \& Miller, 2002). Indeed, there is a rich literature linking social explanations to justice concerns (see Hoffman \& Hurst, 1990; Jost \& Banaji, 1994; Lerner, 1980; Reyna, Henry, Korfmacher, \& Tucker, 2005; Weiner, 2006; Yzerbyt, Rocher, \& Schadron, 1997), although the studies below will examine facets of this link that have received little attention. The justice motive refers to the desire to view the world as just or fair, in the sense that the treatment accorded to people is commensurate with their 'deservingness'. The justice motive reflects the desire to believe that 'good things happen to good people' and 'bad people get punished'. Existing literature supports the notion that the justice motive is deeply ingrained in people's value systems (Lerner, 1980; M. Ross \& Miller, 2002), and thus behaving out of line with its dictates should foster compunction (Higgins, 1987).

Critically, social explanations shape people's reasoning about justice. This connection was initially made clear by Hoffman and Hurst (1990). They argued that, rather than forming stereotypes simply by observing the behavior of different groups, people construct stereotypes that make the social status quo (e.g. division of labor; social stratification) seem fair. For Hoffman and Hurst, then, stereotypes function as internal social explanations: they refer to internal qualities of a group (e.g. kind, gentle) that offer a ready-made explanation for the group's social status and action (e.g. 'Of course they take care of the children-they are kind and gentle!'). Indeed, building on Hoffman and Hurst, numerous scholars have converged on a critical point: negative orientations toward a group can be seen as perfectly just if internal explanations are created that rationalize that negative orientation (e.g. Jost \& Banaji, 1994; Reyna et al., 2005; Sidanius \& Pratto, 1999; Yzerbyt et al., 1997). In other words, employment discrimination is fair if a group is perceived as lazy, limited social roles are fair if a group is perceived as 'naturally suited' for such roles, and even hostility is fair if a group is perceived as having morally undesirable qualities. Because internal explanations justify negative orientations toward a group, we do not expect those who embrace internal explanations for the negative social status of African Americans to experience prejudice-related compunction. Such perceivers associate African Americans with a host of undesirable personal qualities, and thus will feel that negative thoughts and feelings toward African Americans are perfectly fair.

What about those who embrace external explanations? External explanations and their implications for intergroup orientations have received limited attention in the literature. We propose that external explanations dramatically shift people's reasoning about justice, and hence shift the likelihood that people will experience compunction in response their own biases. To elaborate, individuals invoking external explanations focus on factors 'outside of' a group as giving rise to the status or action of the group. Because of this, the internal qualities of the group are of unknown-and perhaps high-desirability. Indeed, from within an external framework, the type of treatment or social position that a group 'truly deserves' cannot be inferred from the group's current social status or action because these were determined by factors outside of the group per se. By analogy, just as one cannot know the skill of a drummer who is wearing handcuffs, one cannot know the characteristics and potentialities of a group that has been 'handcuffed' by discriminatory social policies, fear of more powerful or more heavily armed groups, lack of economic opportunities, and so on. Therefore, from within this explanatory mindset, any negative behavior or thinking toward the group is a violation of the justice motive because such thought and behavior is incommensurate with the perceived deservingness of the group. In fact, negative thinking or behavior might even be viewed as 'adding insult to injury', given that the group is already seen as enduring unearned suffering. Because negative orientations toward a group are a violation of the justice motive for those embracing external social explanations, 
we expect such individuals to experience compunction upon becoming aware of their biases toward a group.

\section{The present studies}

The studies below were designed to investigate the relation between social explanations and prejudice-related compunction. To ensure comparability with past work, Study 1 will use materials from Devine et al. (1991). The key prediction is that among those who strongly embrace external explanations there will be a strong, positive association between prejudicerelated discrepancies and compunction. In contrast, we expect that the association between discrepancies and compunction will be weaker among those who weakly embrace external explanations. The rationale for this prediction is derived from our arguments above: among those who strongly embrace external explanations, as their prejudice-related discrepancies increase in size (i.e. as they admit to greater and greater divergence between how they should versus would respond) they will perceive their biases as increasingly unjust and hence will experience compunction. In contrast, for those weakly embracing external explanations, discrepancies will not be perceived as especially unjust and thus there will be a weaker tendency for discrepancies to foster compunction.

Study 2 will utilize a novel paradigm to provide a conceptual replication of Study 1. Participants will first be induced to admit that they would discriminate against Black males (by avoiding them in a 'bad neighborhood'). Then, their reactions-including compunction-to this imagined act of discrimination will be measured. The key prediction is the same as for Study 1: external explanations will be associated with heightened compunction, and the reason is that bias seems more unjust to those who strongly embrace external explanations. One advantage of Study 2 is that we will include a direct measure of the perceived justice of one's bias, thereby enabling a test of our idea that justice concerns mediate the link between external explanations and compunction. Study 2 will also examine another potential mediator: the extent to which bias is seen as 'rational behavior'. Some scholars have suggested that bias need not elicit self-criticism because, for example, avoiding groups that are statistically associated with negative behavior is simply rational (e.g. D'Souza, 1995). To test whether social explanations affect compunction by altering the perceived rationality of bias (e.g. perhaps endorsement of external explanations makes bias seems less rational, hence creating more compunction for having behaved 'irrationally'), Study 2 will also include direct measures of the perceived rationality of one's bias. We do not expect this measure to be as strong a mediator of the link between external explanations and compunction as are justice concerns. The reason is that we do not think that social explanations are particularly relevant to the perceived rationality of bias: for example, an act of discrimination can be seen as rational (e.g. it 'makes sense' to avoid a potentially dangerous encounter) regardless of one's understanding of the causes necessitating that discrimination (e.g. those teenagers are 'evil' vs. those teenagers had the misfortune of being born into a neighborhood filled with violence and neglect).

Although the primary aim of the present article is to examine the relation of social explanations to prejudice-related compunction, we think it is important not to ignore existing work on the role of internal motivation in fostering compunction (Plant \& Devine, 1998). So, in addition to our primary goal of providing support for the social explanations framework, we have a subordinate goal of examining whether social explanations and internal motivation are independent bases of prejudice-related compunction.

\section{Study 1}

\section{Method}

Overview Participants completed a pretest questionnaire gauging their endorsement of internal and external social explanations for African American socioeconomic status. Later in the semester, they returned for an ostensibly unrelated study during which they reported should and would judgments concerning encounters with African Americans as well as 
their emotional reactions to any discrepancies between their should and would judgments. The key prediction was that prejudice-related discrepancies would be more strongly linked to compunction among those strongly as compared to weakly endorsing external social explanations.

Participants Participants who completed both the pretest and follow-up comprised 63 White female and 58 White male undergraduates at Lehigh University. All participants participated for course credit in their introductory psychology course.

\section{Procedure}

Pretest Altogether, 136 White Lehigh undergraduates (67 female) attended pretest sessions in groups of 2 to 20 . After providing informed consent, they completed numerous questionnaires concerning their personalities and social attitudes. Of relevance to the current study, they completed the Social Explanations Questionnaire (SEQ; Gill \& Andreychik, 2006; see Appendix A) and the Internal and External Motivations Scale (I \& EMS; Plant \& Devine, 1998). For reasons that will become clear below, our final sample included 99 participants (53 female). All descriptive statistics below are based on that final sample.

The SEQ comprised 18 items that gauged the extent to which the respondent endorsed particular causal frameworks for understanding African American socioeconomic status. Twelve of the items tapped internal explanations, i.e. explanations that focus on features ostensibly inherent in African Americans. Six of the items tapped external explanations, i.e. explanations that focus on factors in the environment or circumstances of African Americans. Participants rated each item on a 6-point scale ranging from Disagree strongly (1) to Agree strongly (6). Based on pilot research suggesting that internal and external items load on separate factors in a factor analysis, separate scores were calculated for each by averaging the 12 internal items $(M=2.01$, $S D=.81 ; \alpha=.92)$ and the six external items $(M=4.10, S D=.95 ; \alpha=.82)$. In the present sample, the correlation between internal and external explanations was modest $(r(97)=-.25$, $p=.01)$.

The I\&EMS (Plant \& Devine, 1998) comprises five items measuring internal motivation (e.g. 'I attempt to act in nonprejudiced ways toward Black people because it is personally important to me') and five items measuring external motivations (e.g. 'Because of today's PC-politically correct-standards I try to appear nonprejudiced toward Black people'). Participants responded to all 10 items using a scale with endpoints labeled Strongly disagree (1) and Strongly agree (9). Based on Plant and Devine's scoring procedure, we averaged the five internal motivation items to create an IMS score $(M=6.00, S D=1.24 ; \alpha=.90)$ and we averaged the five external motivation items to create an EMS score $(M=5.05, S D=1.20 ; \alpha=.86)$.

Follow-up Participants who had completed the pretest were invited to participate in the followup, which occurred approximately one month later. Altogether, 120 participants attended survey sessions in groups of 2 to 15 and were not informed of the relation between the pretest and the follow-up. Both the location and experimenter were different from the pretest. Upon arrival, they read a consent form describing a study of 'attitudes on potentially controversial issues, including racial issues'. After signing the consent form, they were given a survey packet that contained the measures described below along with some filler questionnaires.

Participants first reported their personal standards for how they should respond in five situations involving Black people (see Devine et al., 1991). An example is: 'Imagine that a Black person boarded a bus and sat next to you. You should feel uncomfortable that a Black person is sitting next to you'. For each situation, participants circled a number on a scale ranging from Strongly disagree (1) to Strongly agree $(7)$. After appropriate reverse scoring such that higher scores indicated more prejudiced should ratings, a total should score was formed by averaging ratings across the five situations $(M=1.77, S D=1.06 ; \alpha=.77)$.

After completing the should ratings, participants provided responses regarding how they would 
actually respond in the same five situations (e.g. 'Imagine that a Black person boarded a bus and sat next to you. You would feel uncomfortable that a Black person is sitting next to you'). For each situation, participants circled a number on a scale ranging from Strongly disagree (1) to Strongly agree (7). After appropriate reverse scoring such that higher scores indicated more prejudiced would ratings, a total would score was formed by averaging ratings across the five situations $(M=2.32, S D=1.28 ; \alpha=.78)$.

A discrepancy index was calculated by subtracting participants' should ratings from their would ratings for each situation and then averaging across the five situations. This resulted in an index on which positive scores indicate that one's predicted actual (would) responses are more prejudiced than her desired (should) responses. On this index, $42 \%$ of participants showed a positive discrepancy, $41 \%$ showed no discrepancy, and $17 \%$ showed a negative discrepancy. Following Devine et al. (1991) and Monteith et al. (1993), our analyses below involve only participants with zero or positive discrepancy scores (i.e. we excluded the $17 \%$ of participants who said they would behave less prejudicially than they should). This resulted in 99 usable cases $(M=.55, S D=.86 ; \alpha=.75)$.

After completing should and would ratings, participants reported their feelings regarding any discrepancies between these ratings (e.g. how they felt about the fact that they would behave more prejudicially than they think they should). They indicated on a scale ranging from Does not apply at all (1) to Applies very much (7) the degree to which each of 35 affect terms described their feelings. The affect items were the same as those used in prior research (e.g. Devine et al., 1991; Monteith et al., 2002).

Following data collection, the affect items were combined as in Monteith et al. (2002). That is, we created indices of: compunction (angry at myself, guilty, annoyed with myself, regretful, disappointed with myself, disgusted with myself, shameful, selfcritical $M=2.28, S D=1.60 ; \alpha=.97)$; discomfort (fearful, uneasy, embarrassed, anxious, threatened, frustrated, bothered, uncomfortable, tense, $M=2.16$, $S D=1.34 ; \alpha=.94$ ); other-directed negativity (angry at others, disgusted with others, irritated with others,
$M=2.22, S D=1.45 ; \alpha=.85) ;$ and positive emotions (energetic, friendly, good, optimistic, happy; $M=3.38$, $S D=1.44 ; \alpha=.80)$. The indexes of other-directed negative emotions and positive emotions yielded nothing of theoretical interest. That is, each was predicted only by discrepancies (i.e. larger discrepancies produced more other-directed negative emotion and less positive emotion). Thus, those indexes will not be discussed further.

\section{Results}

Our primary interest was in predicting people's emotional reactions-especially compunctionto their prejudice-related discrepancies. To test our hypotheses, we used multiple regression analysis and, in all cases, centered variables on their means before multiplying them to create interaction terms (Cohen \& Cohen, 1983).

We first regressed compunction on discrepancies, external explanations, internal explanations, IMS, EMS, and the interactions of these latter four variables with discrepancies. ${ }^{3}$ This analysis revealed just two significant effects. First, there was a main effect of discrepancies, $(t(85)=4.27$, $p=.00005 ; \beta=.44)$, such that compunction increased as the size of discrepancies increased. This main effect is found in virtually all studies using the should-would measures and suggests that, in general, people experience compunction when they imagine that they would behave more negatively than they should. The critical question for us was whether the strength of the relation between discrepancies and compunction was moderated by external explanations, such that those embracing external explanations show a particularly strong tendency to experience compunction. Indeed, as predicted, there was an interaction between external explanations and discrepancies, $(t(85)=2.70, p=.008 ; \beta=.26)$. To explore the nature of this interaction, we conducted simple slope analyses (Aiken \& West, 1991) using the ModGraph program (Jose, 2002). These examined the relation between discrepancies and compunction at varying levels of endorsement of external explanations. As can be seen in Figure 1, these analyses revealed the predicted pattern: discrepancies were strongly associated with compunction among those 
who strongly or moderately endorsed external explanations (both $t \mathrm{~s}(95)>4.98$, $p \mathrm{~s}<.000003$ ). In contrast, discrepancies were only marginally associated with compunction among those who weakly endorsed external explanations $(t(95)=1.82, p=.07)$. The interaction between IMS and discrepancies in this analysis was weak $(t(85)=1.48, p=.14 ; \beta=.23)$. Before drawing any conclusions about the effects of IMS, however, we must present some further analyses. Finally, the interactions of discrepancies with both internal explanations and external motivation were negligible $(t \mathrm{~s}<1)$.

Next, we regressed discomfort on discrepancies, external explanations, internal explanations, IMS, EMS, and the interactions of these latter four variables with discrepancies. This analysis revealed one significant and one marginal effect. First, as with compunction, there was a main effect of discrepancies $(t(85)=3.80, p=.0003$; $\beta=.42$ ), such that discomfort increased as the size of discrepancies increased. Second, as with compunction, there was an interaction between external explanations and discrepancies $(t(85)=1.95, p=.055 ; \beta=.20)$. This interaction, however, disappeared $(t<1)$ when compunction was added as an additional predictor in the multiple regression model. This supports the possibility that external explanations are not relevant to discomfort per se, but rather moderate the relation between discrepancies and discomfort only because discomfort shares some association with compunction.

But, are external explanations relevant to compunction per se? That is, do external explanations create a specific tendency for discrepancies to foster compunction above and beyond any association that compunction shares with discomfort? To examine this issue, we repeated the regression analysis in which compunction was regressed on discrepancies, external explanations, internal explanations, IMS, EMS, and the interactions of these latter four variables with discrepancies. In this follow-up analysis, however, we added discomfort as another predictor. Critically, even with discomfort added to the model the interaction between external explanations and discrepancies remained significant $(t(84)=2.04, p=.04)$, suggesting that external explanations are specifically relevant to the tendency for discrepancies to foster compunction above and beyond any association that compunction shares with discomfort.

Interestingly, in the preceding analysis controlling for discomfort, the interaction between discrepancies and IMS—as found by Plant and

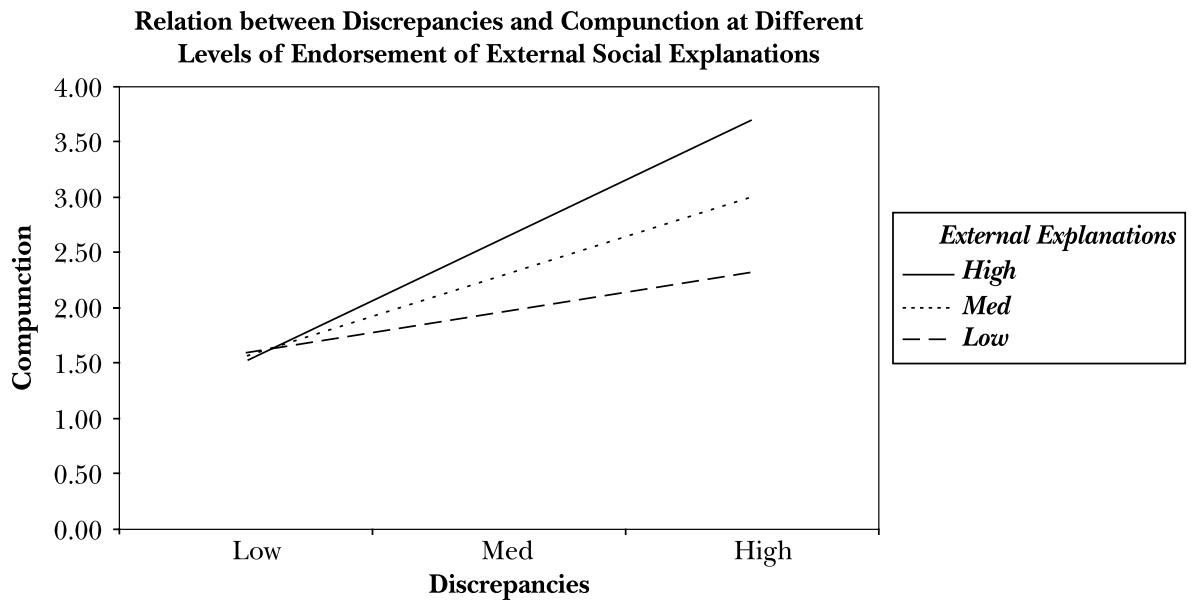

Figure 1. Simple slope analysis revealing that the relation between discrepancies and compunction is moderated by endorsement of external social explanations. As per Aiken and West (1991), high level of endorsement is $1 S D$ above the mean, medium is the mean, and low is $1 S D$ below the mean. 
Devine (1998)—became statistically significant. To examine the nature of this interaction, we first computed residual compunction scores after controlling for discomfort, external explanations, internal explanations, EMS, and the interactions of these latter three variables with discrepancies. ${ }^{4}$ Then, following Aiken and West (1991), we conducted simple slope analyses examining the relation between discrepancies and residual compunction at varying levels of IMS (using the Modgraph program; Jose, 2002). These analyses revealed a pattern similar to that uncovered by Plant and Devine: discrepancies were strongly and positively associated with residual compunction among those high (i.e. $+1 S D$ from the mean) or medium (i.e. at the mean) in IMS (both $\beta \mathrm{s}>.24, p \mathrm{~s}<.05$ ), but unrelated to residual compunction among those low (i.e. $-1 S D$ from the mean) in IMS $(t<1)$. Because this interaction of IMS with discrepancies was significant in a multiple regression that also included the interaction of external explanations and discrepancies, these data support the possibility that social explanations and IMS are independent bases of prejudice-related compunction.

This recognition raises an important issue: do social explanations predict compunction above and beyond what can be predicted from variables previously explored in the literature? That is, does the social explanations concept add anything new to our understanding? The preceding paragraph suggests an affirmative answer to this question, at least with respect to IMS. In addition to IMS, though, prior work (Devine et al., 1991; Monteith, 1993) has also linked global prejudice to compunction, with those low in global prejudice experiencing more compunction in response to discrepancies than those high in global prejudice. Do social explanations predict compunction above and beyond what can be predicted from global prejudice? Unfortunately, we did not administer any well-known measures of global prejudice.

Yet, it is worth noting that IMS is strongly linked to such measures, showing very strong correlations $(r=.66)$ with Brigham's Attitudes toward Blacks Scale (Devine, Plant, Amodio, Harmon-Jones, \& Vance, 2002). Thus, our findings regarding IMS indirectly suggest that social explanations might have effects independent of global prejudice levels. Of even greater relevance, our measure of internal explanations seems quite similar to traditional measures of prejudice, as it includes items concerning belief in inherent inferiority of African Americans, association of African Americans with negative traits, and so on. Thus, the fact that the interaction between external explanations and discrepancies was significant even when internal explanations and their interaction with discrepancies were statistically controlled strongly supports the notion that the effects of external explanations are not explicable in terms of global prejudice levels.

\section{Discussion}

Study 1 supported our expectation that social explanations are associated with the experience of prejudice-related compunction. More specifically, as predicted, the tendency to experience compunction in response to prejudice-related discrepancies was quite pronounced among people who strongly embraced external explanations for African American socioeconomic patterns, but weaker among those who weakly embraced external explanations. Furthermore, external explanations were specifically relevant to compunction. That is, they were associated with compunction in response to discrepancies even when discomfort was statistically controlled, and not associated with discomfort in response to discrepancies when compunction was statistically controlled. In addition, Study 1 also suggested that social explanations provide novel information about the basis of prejudice-related compunction. That is, the moderating effect of external explanations on the relation between discrepancies and compunction remained evident even after statistically controlling for effects involving IMS and global prejudice (as measured by our internal explanations scale).

We must also acknowledge limitations of Study 1. In particular, the should and would judgments involve hypothetical scenarios. Thus, it is possible that people's responses to them are not relevant to reactions to 
real-world interracial encounters. For example, whereas some people reported compunction in response to imagining discomfort in the presence of a Black person, those people might not actually experience compunction if such discomfort actually occurred. If this were true, one reason might be that people engage in stronger self-justification efforts when it comes to their actual behavior (i.e. they might strive harder to convince themselves they are a good person by justifying their biases). On the other hand, though, it is also plausible that the use of hypothetical scenarios underestimates the extent to which people experience compunction. That is, whereas one might feel a little compunction upon imagining discomfort in the presence of a Black person, one might feel a lot of compunction upon experiencing discomfort in the presence of a flesh-and-blood fellow human being (i.e. 'How could I treat a person in this way!?'). Finally, it is worth nothing that Monteith and her colleagues (1993; Monteith et al., 2002) have studied prejudice-related compunction and self-regulation using somewhat more realistic scenarios and found results that closely parallel those based on the hypothetical should/would paradigm. They have also provided substantial evidence for the validity of the type of discrepancy measure used in Study 1 (Monteith \& Voils, 1998).

\section{Study 2}

Study 2 will use a novel set of research materials to examine the phenomenon of prejudicerelated compunction. The approach will involve inducing participants to admit that they would discriminate under certain conditions and then having them report how they would feel about this act of discrimination. Specifically, we created a vignette in which one's nonprejudiced standards would conflict with one's standards based on personal safety: Participants were asked whether they would avoid Black male teenagers in a 'bad neighborhood'. We expected that most participants would make a decision based on personal safety concerns, but that some of these participants would nevertheless experience compunction regarding their decision. As above, our prediction was that those strongly embracing external explanations would be most likely to experience prejudice-related compunction.

Furthermore, as mentioned above, Study 2 will also examine mediators of the effect of social explanations on compunction. Above, we argued that social explanations modify the extent to which bias is perceived as just or fair, and that compunction grows out of perceptions of (in)justice. Therefore, in Study 2, we will include a direct measure of beliefs about the justness/fairness of one's discriminatory action. In addition, as described above, Study 2 will also include a measure of perceptions of the rationality of one's discriminatory action. This will enable a test of whether social explanations influence compunction because they modify the extent to which prejudice is seen as rational behavior. Whereas we have a strong prediction that justice/fairness perceptions will mediate the effect of social explanations on compunction, our predictions regarding rationality perceptions are less certain (see above).

\section{Method}

Overview During a pretest, participants completed the same measures of social explanations and internal and external motivations as in Study 1. Weeks later, participants completed a measure asking whether they would discriminate by avoiding a group of Black teenagers in a 'bad neighborhood'. Among those who answered 'yes', items tapping compunction, emotional distress, belief in the justice/fairness of their action, and belief in the rationality of their action were presented. The key predictions were that external explanations would be linked to the experience of prejudice-related compunction (as in Study 1) and furthermore that beliefs about the fairness of one's discriminatory action would mediate this link.

Participants Sixty-two White female and 58 White male undergraduates at Lehigh University participated for credit in their introductory psychology course. 


\section{Procedure}

Pretest At the beginning of the semester, participants took part in a pretest. After signing a consent form indicating that they would complete some potentially controversial measures of racial attitudes, they completed the SEQ. As in Study 1, each participant received an external explanations score $(M=4.09 ; S D=1.02 ; \alpha=.83)$ and an internal explanations score $(M=2.06$, $S D=.85 ; \alpha=.90)$. The correlation between these scores in the present sample was $(r(118)=-.29$, $p=.002)$. Participants also completed Plant and Devine's (1998) I\&EMS. As in Study 1, separate scores were computed for IMS $(M=6.83, S D=$ $1.81 ; \alpha=.90)$ and EMS $(M=5.12, S D=1.96$; $\alpha=.86)$.

Follow-up A few weeks after the pretest, participants were asked to participate in the follow-up phase of the study. Participants were not made aware of the relation between the two phases, and each phase had a different experimenter and took place at a different location. During the follow-up, after completing a Consent Form indicating that the study would concern potentially controversial racial attitudes, participants completed a measure asking whether they might try to avoid Black male teenagers in a bad neighborhood (see Appendix B). Fully $89.2 \%$ of participants $(N=107)$ indicated that they would. ${ }^{5}$

Crucially, the measure also solicited-from the 107 participants who said they might practice avoidance-the respondent's reaction to his or her imagined avoidance (see, again, Appendix B). Ratings on each item were made on a scale ranging from Feel this not at all (1) to Feel this strongly (6). Because prior work has distinguished between compunction and other types of negative emotions, we included two items tapping compunction and three items tapping emotional distress other than compunction. Furthermore, to test our hypotheses regarding potential mediators, we included one item concerning perceptions of the justice of one's discriminatory behavior and a few items concerning the extent to which one thinks one's discriminatory behavior is rational. After appropriate reverse scoring, we combined the items shown in Appendix B to form indexes of compunction $(M=3.43, S D=1.48 ; \alpha=.72)$, emotional distress other than compunction $(M=3.42, S D=1.20 ; \alpha=.69)$, belief in the justice of one's discriminatory act $(M=2.75$, $S D=1.49)$, and belief in the rationality of one's discriminatory act $(M=4.40, S D=0.98$; $\alpha=.77)$.

\section{Results}

Our primary interest was in predicting who experienced compunction in response to their imagined act of discrimination. We examined this among the 107 participants who had answered that they might engage in such discrimination. In addition, we aimed to test two hypotheses regarding mediation: (a) social explanations influence compunction because they affect the extent to which negative reactions to a group are perceived as fair and just; and (b) social explanations influence compunction because they affect the extent to which discrimination is viewed as rational behavior.

Our primary analysis involved regressing the compunction index on external explanations, internal explanations, IMS, and EMS. This revealed a significant effect of external explanations $(t(102)=3.17, p=.002 ; \beta=.32)$, the direction of which suggested that those who strongly embraced external explanations reported stronger feelings of compunction than did those who weakly embraced external explanations. The effect of IMS was in the expected direction, but weak $(t(102)=1.46$, $p=.147 ; \beta=.18)$. The effects of internal explanations and EMS were negligible (both ts $<1.27, p \mathrm{~s}>.20)$. Notably, this tendency for external explanations to be particularly potent for predicting compunction replicates the pattern found in Study 1.

Do the present data also confirm the finding of Study 1 that external explanations are particularly relevant for compunction but not other forms of emotional distress? To examine this, we regressed compunction on external explanations, internal explanations, IMS, EMS, and emotional distress. The effect of emotional distress was highly significant $(t(101)=7.11$, $p<.00001 ; \beta=.57)$, indicating a strong positive 
association between compunction and other types of emotional distress. Yet, in this analysis the relation between external explanations and compunction remained significant $(\mathrm{t}(101)=2.07$, $p=.041)$. Furthermore, we found that when emotional distress was regressed on external explanations, internal explanations, IMS, and EMS, the only significant effect was that of external explanations $(t(102)=2.47, p=.015$; $\beta=.25)$. Yet, when we added compunction as a predictor in this regression model, the effect of external explanations on emotional distress disappeared $(t<1)$. Taken together, these analyses are congruent with the findings of Study 1 in suggesting that external explanations are specifically relevant to compunction and not to other types of emotional distress.

Next, we turned our attention to potential mediators: justice concerns and perceptions of rationality. To test mediation, we followed the procedures outlined by Baron and Kenny (1986). As can be seen in Table 1, there were significant zero-order relations between external explanations and compunction and between external explanations and both potential mediators (i.e. external explanations were associated with heightened justice concerns and diminished perceptions of rationality). Thus, mediation is a possibility for both potential mediators. To directly test mediation, we computed multiple regression analyses that enabled us to see the extent to which the beta weight representing the effect of external explanations on compunction was reduced when each mediator was separately added to the regression model. As can be seen in the top part of Table 1, the beta weight representing the effect of external explanations was reduced by approximately $50 \%$ when justice concerns were added as a predictor in the multiple regression model. This indicated significant mediation, as confirmed by a Sobel test (see Table 1). In addition, as can be seen in the lower part of Table 1, in a separate multiple regression analysis, the beta weight representing the effect of external explanations was reduced by approximately $20 \%$ when belief in the rationality of one's discrimination was added as a predictor in the multiple regression model. This represented a marginal mediation effect, as suggested by a Sobel test

Table 1. Regression analyses testing mediating roles of justice concerns and perceptions of the rationality of discrimination (Study 2)

\begin{tabular}{|c|c|c|c|c|}
\hline Outcome variable & Predictor variable & $\beta$ & $t$ & $p$ \\
\hline \multicolumn{5}{|l|}{ Justice concerns as mediator } \\
\hline Eq. 1: Compunction & External explanations & .38 & 4.16 & .0001 \\
\hline Eq. 2: Justice concerns & External explanations & .29 & 3.09 & .003 \\
\hline Eq. 3: Compunction & External explanations & .20 & 2.72 & .008 \\
\hline & Justice concerns & .61 & 8.23 & $<.0001$ \\
\hline \multicolumn{5}{|c|}{ Statistical significance of mediation } \\
\hline \multicolumn{2}{|c|}{ Drop in external explanations beta } & & & \\
\hline Sobel test & \multicolumn{2}{|c|}{$2.91, p=.004$} & & \\
\hline \multicolumn{5}{|c|}{ Perceptions of rationality as mediator } \\
\hline Eq. 1: Compunction & External explanations & .38 & 4.16 & .0001 \\
\hline $\begin{array}{l}\text { Eq. 2: Perceptions of } \\
\text { rationality }\end{array}$ & External explanations & -.21 & -2.23 & .03 \\
\hline \multirow[t]{2}{*}{ Eq. 3: Compunction } & \multirow{2}{*}{$\begin{array}{l}\text { External explanations } \\
\text { Perceptions of rationality }\end{array}$} & .30 & 3.51 & .001 \\
\hline & & -.34 & -3.91 & .0002 \\
\hline \multicolumn{5}{|l|}{ Test of mediation } \\
\hline \multicolumn{2}{|c|}{ Drop in external explanations beta } & & & \\
\hline Sobel test & \multicolumn{2}{|c|}{$1.90, p=.06$} & & \\
\hline
\end{tabular}


(see Table 1). Thus, consistent with our expectations, the strongest evidence regarding mediation is that external explanations seem to foster compunction because they heighten concerns about the fairness of biases. ${ }^{6}$

Finally, as in Study 1, we examined whether social explanations predict compunction above and beyond what can be predicted from variables previously explored in the literature. Data from Study 2 have already suggested that external explanations predict compunction above and beyond what can be predicted from IMS. What about global prejudice? In Study 2, our pretest included a measure of positive attitudes toward African American culture (created by the authors).$^{7}$ This is perhaps better thought of as a measure of positive global attitudes than as a measure of global prejudice (assuming that positive and negative attitudes are not simply the inverse of each other; see Katz \& Hass, 1988). As might be expected, this measure showed a positive zero-order correlation with compunction $(r(105)=.19, p=.05)$, suggesting that those who have positive attitudes toward African American culture experience more compunction in response to imagined discrimination. Importantly, however, when we regressed compunction on positive attitudes toward African American culture, external explanations, internal explanations, IMS, and EMS, the effect of external explanations remained significant $(t(101)=3.30$, $p=.001)$. Replicating Study 1, this suggests that social explanations provide novel information about the foundations of prejudice-related compunction. The effect of positive attitudes toward African American culture, however, became nonsignificant $(t<1)$ in this analysis.

\section{Discussion}

Study 2 looked at prejudice-related compunction using a novel paradigm. The findings were remarkably consistent with Study 1 and, in addition, added important new information about mediation. Participants were asked whether they would engage in discriminatory behavior with respect to young Black males in a 'bad neighborhood'. Almost $90 \%$ of respondents said that they would. We examined people's emotional and cognitive reactions to this imagined discrimination. Conceptually replicating Study 1, Study 2 revealed that those who strongly embrace external explanations experience more compunction in response to their biases than do those who weakly endorse external explanations. Also, just as in Study 1, external explanations were specifically relevant to compunction and not to other types of emotional distress. Critically, Study 2 also provided mediational evidence consistent with our argument in the Introduction. That is, there was a significant mediational effect consistent with the notion that external explanations foster compunction because they increase the extent to which negative orientations toward a group seem unjust or unfair. Furthermore, we found weaker mediational evidence consistent with the notion that external explanations foster compunction because they decrease the extent to which negative orientations seem rational.

Finally, Study 2 also provided evidence that the effects of external explanations on compunction are independent of IMS, but the evidence was more equivocal than in Study 1. In particular, the effect of external explanations was significant when IMS was included in the multiple regression model, but the effect of IMS was weak. Given that the relevance of IMS to compunction has been demonstrated in several studies (our Study 1; Plant \& Devine, 1998), it is probably reasonable to attribute this weak result to sampling error. Finally, Study 2 also provided evidence that external explanations have effects above and beyond the effects of global positive evaluations of African Americans. That is, despite the fact that positive attitudes toward African American culture showed a significant zero-order correlation with compunction, the effect of external explanations on compunction remained significant even when positive attitudes toward African American culture were included in the multiple regression model.

\section{General discussion}

The literature suggests that many people hold ambivalent intergroup attitudes, showing prejudicial responses that they simultaneously consider unacceptable (e.g. Allport, 1954; 
Devine et al., 1991; Monteith, 1993; Monteith et al., 2002). The literature also suggests that those who experience compunction in response to their undesired biases are particularly likely to subsequently engage in self-regulatory strategies that will reduce their biases in both the shortand long-term (e.g. Monteith, 1993; Monteith et al., 2002). Because of the importance of compunction in energizing these positive forms of self-regulation, it is crucial to understand the psychological foundations of prejudice-related compunction.

We sought to apply a social explanations framework to this issue. The social explanations framework centers on the idea that perceivers seek to explain group-based patterns in society (Why are some groups poor? Why are some groups violent?), and that the particular explanatory frameworks they create or embrace have implications for their subsequent orientation toward a group. In the present article we have focused on external explanations, which locate the cause of a group's outcomes or actions "outside of" the group members and which imply that any group exposed to similar external causal forces would show similar outcomes or actions (see Gilbert, 1998; L. Ross, 1977).

In particular, we hypothesized that those who embrace external explanations regarding the negative status and action of a group will be more likely to experience prejudice-related compunction. We reached this hypothesis through a consideration of work on the justice motive (Lerner, 1980; M. Ross \& Miller, 2002). We reasoned that one who believes that a low status group has been 'pushed around' or 'held down' by external forces will feel compunction if she shows signs of a negative orientation (i.e. a prejudicial thought, feeling, or behavior) toward that group. The reason is that, from her perspective, the negative orientation is unjust because it involves bias toward a group whose negative status or action was substantially molded by forces outside of the group. Hence, the group does not clearly deserve to be on the receiving end of negative thoughts, feelings, or behaviors.

Our predictions regarding the relation between external explanations and prejudice-related compunction were supported in both Study 1 (using Devine et al.'s should-would paradigm) and Study 2 (using a novel paradigm that involved imagining one's self discriminating against Black males). That is, compared to those who weakly embrace external explanations, those who strongly embrace external explanations for African American socioeconomic patterns were particularly likely to experience compunction in response to prejudice-related discrepancies involving African Americans (Study 1) and in response to imagining themselves discriminating against Black males (Study 2). In addition, the data suggested that external explanations are specifically relevant to compunction and do not show a specific tendency to foster other types of negative emotions. Finally, the data from Study 2 were also consistent with our proposed mediational model: external explanations foster compunction because such explanations increase concerns about the justice of having a negative orientation toward a group.

These findings contribute to the literature concerning bases of prejudice-related compunction. As noted above, both global prejudice (Devine et al., 1991) and IMS (Plant and Devine, 1998) have been linked to prejudice-related compunction. Importantly, our data suggested that the effects of external explanations are not explicable in terms of these other constructs. Indeed, the effect of external explanations on compunction remained statistically significant even after we statistically controlled for internal explanations (which we interpret as an indicator of global prejudice; Studies $1 \& 2$ ), the interaction of internal explanations with discrepancies (Study 1), IMS (Studies $1 \& 2$ ), and global positive evaluations of African Americans (Study 2). Notably, we also found evidence that the effects of social explanations and IMS are somewhat independent. That is, both predictors were related to compunction in regression models that controlled for the effect of the other predictor (although the effects of social explanations were somewhat more robust). How can we understand, at a conceptual level, this independence between social explanations and IMS? One possibility is that, as proposed above, social explanations relate to thinking about social justice: what kind 
of society have we created and how must we act to make it the kind of society we want it to be? In contrast, IMS might have more to do with a personal emphasis on being a kind person. This possibility is suggested by Plant and Devine's (1998) data, which showed a strong correlation between IMS and humanitarian values. Future research, of course, is necessary to test these speculations (although Study 2 did provide evidence that social explanations are relevant to justice concerns).

Other work from our lab is also relevant to the issue of the divergent validity of social explanations vis-a-vis other facets of intergroup attitudes. Of greatest relevance, Gill and Andreychik (2006; Study 1) found that social explanations regarding African Americans were significantly associated with attitudes toward social policies implicating African Americans even after controlling for the effects of trait stereotypes, feeling thermometer ratings, Modern Racism (McConahay, 1986), Social Dominance Orientation (Sidanius \& Pratto, 1999), and Right-Wing Authoritarianism (Altemeyer, 1998). Taken together with the present studies, this work strongly suggests that the social explanations framework is not merely redundant with existing conceptual approaches to intergroup attitudes.

Our findings in the present article underline the crucial role of external explanations. This is important because, with few exceptions (e.g. Guimond et al., 1989; Lopez et al., 1998), we find that little attention has been paid to such explanations in the intergroup attitudes literature. Indeed, even the work that has been done focuses on the existence of external explanations (see Guimond et al., 1989; Lopez et al., 1998) rather than on how such explanations might shape other aspects of intergroup cognition, emotion, and behavior. In contrast, internal explanations have received the lion's share of attention. This becomes more evident when one considers that ubiquitously studied stereotypes are often a type of internal explanation (e.g. the stereotype of African American 'laziness' is an internal explanation for the low social position of African Americans; Allport, 1954; Jost \& Banaji, 1994). We must add the caveat here that our data do not, of course, uniquely support a causal role of external explanations because our data are correlational in nature. As discussed in the introduction, however, other work from our lab (Gill \& Andreychik, 2006; Study 4) comes closer to providing evidence for such a causal role.

Finally, we think it is significant to note how the social explanations framework builds a bridge between work on intergroup emotion and work on emotion in other domains. Diverse areas of study are unified by the notion that the psychological process of explanation is a foundation of emotional experience. Weiner (1986, 2006) has been particularly prolific in linking explanation to emotion. For example, he has highlighted how explanations impact self-directed emotions (e.g. self-esteem), other-directed emotions (e.g. anger, gratitude), and emotions triggered by outcomes (e.g. a given success or failure). Similar to Weiner's focus is Grant and Dweck's (2003) work suggesting that whether one explains difficult experiences in the classroom in terms of an entity or incremental framework determines whether one is devastated or energized by such difficulties. Furthermore, a large body of work has grown up around the finding that depressive emotions are rooted in particular styles of causal explanation (e.g. Buchanan \& Seligman, 1995). Finally, emotional reactions to illness also appear to be rooted in how one explains the illness (see Michela and Wood, 1986, for a review). The present work thus unites work on intergroup emotion with work on emotion in other domains by showing that, across these diverse areas, emotional experience has a common foundation in the types of explanatory frameworks employed by individuals striving to understand their worlds.

\section{Notes}

1. We share Gilbert's (1998; see also L. Ross, 1977) interpretation of external causation. Strictly speaking, external forces cannot directly cause behavior. Yet, people often point to external forces when they explain behavior: 'He had a rough childhood'. We agree with Gilbert that what these external explanations mean is this: 'His characteristics are the characteristics 
that anyone would have if subjected to the same experiences'. That is, his thoughts, feelings, and behaviors can be understood as natural and predictable human responses to the experience of having a rough childhood.

2. Of course, there are other dimensions along which explanations can vary. For example, Weiner (1986) offered the dimensions of stable vs. unstable and controllable vs. uncontrollable. Dweck (1999) offered the notion that explanations can refer to malleable (incremental theory) or fixed (entity theory) features of an actor. Malle (2004) offered the idea that explanations tend to characterize behaviors as unintentional or intentional, and, for behaviors characterized as intentional, explanations involve characterizing the actor's reasons or the causal history of those reasons. Despite this conceptual diversity, the application of these frameworks to issues of intergroup cognition and emotion is in its infancy (but see Levy, Stroessner, \& Dweck, 1998; O'Laughlin \& Malle, 2002). Because existing evidence pertains primarily to the roles of internal and external frameworks in the intergroup context (e.g. Guimond et al., 1989; Hewstone, 1990; Kluegel \& Bobo, 1993; Lopez et al., 1998; Pettigrew, 1979; Taylor \& Jaggi, 1974), the present analysis will focus on those. Future research, however, is clearly needed to explore the relevance of the entire gamut of explanatory frameworks for intergroup cognition and emotion.

3. For some analyses, degrees of freedom are lower than would be expected based on the number of participants in our study. This stems from missing data. For example, for the present regression model, data from four participants could not be used. Two of these participants failed to complete the compunction items, one failed to complete the internal explanations items, and one failed to complete the EMS items.

4. The IMS by discrepancies interaction is significant only when controlling for all these other predictors. Thus, examining the IMS by discrepancies interaction using 'raw' compunction scores, i.e. without calculating these residual scores, reveals a pattern that is clearly not a statistical interaction.

5. As noted by a reviewer, this cannot be taken as evidence for racial bias per se because similar levels of discrimination might be observed if the scenario referred to White teenagers in a bad neighborhood. We do not mean to imply otherwise. Nevertheless, our analyses suggest there is a racial component to this scenario because people's emotional and cognitive reactions to discriminating are quite predictable from their social explanations regarding African Americans.

6. Notably, in both cases the mediation effect represents partial rather than full mediation, as indicated by the fact that the relation between external explanations and compunction remained significant when either mediator was added to the multiple regression model. We suspect that an even stronger mediating role of justice concerns would have emerged had we created a better, multi-item measure of that construct. Future research is needed to examine this claim.

7. This consisted of seven items rated on a scale ranging from Strongly disagree (1) to Strongly agree (6). The items were: 'People could learn a lot about social justice and philosophies of social change if they would read the works of great African American social leaders such as Martin Luther King, Jr., W.E.B. DuBois, and others'; 'African Americans have made impressive contributions to our collective culture with their creation of blues and jazz musical styles'; 'I admire the work of great African American writers such as Toni Morrison, Richard Wright, Ralph Ellison, James Baldwin, Nora Zeale Hurston, and others'; 'I think that the cultural contributions of African Americans are inferior to those of European Americans'; 'Because African Americans have suffered so much social discrimination, I think the study of their culture could teach us valuable lessons about how to cope with adversity'; 'Overall, I think there is much value in African American culture'; and 'Nothing important would be learned from studying African American culture'.

\section{Acknowledgments}

Study 2 data are part of an Honors Thesis completed by Sheila Clabby. Sincere thanks are extended to Meghan Gove and Erin Schwartz for their assistance with data collection for both studies.

\section{References}

Aiken, L. S., \& West, S. G. (1991). Multiple regression: Testing and interpreting interactions. Thousand Oaks, CA: Sage. 
Allport, G. W. (1954). The nature of prejudice. Reading, MA: Addison-Wesley.

Altemeyer, B. (1998). The other 'authoritarian personality'. In M. P. Zanna (Ed.), Advances in experimental social psychology (Vol. 30, pp. 47-92). New York: Academic Press.

Bargh, J. A. (1999). The cognitive monster: The case against the controllability of automatic stereotype effects. In S. Chaiken \& Y. Trope (Eds.), Dual process theories in social psychology. New York: Guilford.

Baron, R. M., \& Kenny, D. A. (1986). The moderator-mediator distinction in social psychological research: Conceptual, strategic, and statistical considerations. Journal of Personality and Social Psychology, 51, 1173-1182.

Buchanan, G. M., \& Seligman, M. E. P. (Eds.) (1995). Explanatory style. Hillsdale, NJ: Erlbaum.

Cohen, J., \& Cohen, P. (1983). Applied multiple regression/correlation analysis for the behavioral sciences. Hillsdale, NJ: Erlbaum.

Devine, P. G. (1989). Stereotypes and prejudice: Their automatic and controlled components. Journal of Personality and Social Psychology, 56, 5-18.

Devine, P. G., Monteith, M. J., Zuwerink, J. R., \& Elliott, A. J. (1991). Prejudice with and without compunction. Journal of Personality and Social Psychology, 60, 817-830.

Devine, P. G., Plant, E. A., Amodio, D. M., HarmonJones, E., \& Vance, S. L. (2002). The regulation of explicit and implicit race bias: The role of motivations to respond without prejudice. Journal of Personality and Social Psychology, 82, 835-848.

D'Souza, D. (1995). The end of racism. New York: Free Press.

Dweck, C. S. (1999). Selt-theories: Their role in motivation, personality and development. Philadelphia: Psychology Press.

Fazio, R. H., Jackson, J. R., Dunton, B. C., \& Williams, C. J. (1995). Variability in automatic activation as an unobtrusive measure of racial attitudes: A bona fide pipeline? Journal of Personality and Social Psychology, 69, 1013-1027.

Fiske, S. T., Lin, M., \& Neuberg, S. L. (1999). The continuum model: Ten years later. In S. Chaiken \& Y. Trope (Eds.), Dual-process theories in social psychology (pp. 231-254). New York: Guilford.

Gilbert, D. T. (1998). Ordinary personology. In D. T. Gilbert, S. T. Fiske, \& G. Lindzey, (Eds.), The handbook of social psychology (4th ed.). New York: McGraw Hill.

Gill, M. J., \& Andreychik, M. R. (2006). Yours is to reason why: Social explanations as a foundation of intergroup attitudes. Unpublished manuscript, Lehigh University, Bethlehem, PA.

Glick, P., \& Fiske, S. T. (1996). The ambivalent sexism inventory: Differentiating hostile and benevolent sexism. Journal of Personality and Social Psychology, 70, 491-512.

Gopnik, A. (2000). Explanation as orgasm and the drive for causal knowledge: The function, evolution, and phenomenology of the theory formation system. In F. C. Keil \& R. A. Wilson (Eds.), Explanation and cognition (pp. 299-323). Cambridge, MA: MIT Press.

Grant, H., \& Dweck, C. S. (2003). Clarifying achievement goals and their impact. Journal of Personality and Social Psychology, 85, 541-553.

Guimond, S., Begin, G., \& Palmer, D. L. (1989). Education and causal attributions: The development of 'person-blame' and 'systemblame' ideology. Social Psychology Quarterly, 52, 126-140.

Heider, F. (1958). The psychology of interpersonal relations. New York: Wiley.

Hewstone, M. (1990). The 'ultimate attribution error'?: A review of the literature on intergroup causal attribution. European Journal of Social Psychology, 20, 311-335.

Higgins, E. T. (1987). Self-discrepancy: A theory relating self and affect. Psychological Review, 94, 319-340.

Hoffman, C., \& Hurst, N. (1990). Gender stereotypes: Perception or rationalization? Journal of Personality and Social Psychology, 58, 197-208.

Jose, P. E. (2002). ModGraph: A computer programme to graphically display moderation [Computer software]. Retrieved from http:// www.vuw.ac.nz/psyc/staff/paul-jose/files/ modgraph/modgraph.php.

Jost, J. T., \& Banaji, M. R. (1994). The role of system-justification and the production of false consciousness. [Special Issue: Structure, function, and process]. British Journal of Social Psychology, 33, 1-27.

Katz, I., \& Hass, R. G. (1988). Racial ambivalence and American value conflict: Correlational and priming studies of dual cognitive structures. Journal of Personality and Social Psychology, 55, 893-905.

Keller, J. (2005). In genes me trust: The biological component of psychological essentialism and its relationship to medianisms of motivated social cognition. Journal of Personality and Social Psychology, 88, 686-702.

Kluegel, J. R., \& Bobo, L. (1993). Dimensions of Whites' beliefs about the Black-White 
socioeconomic gap. In P. M. Sniderman, P. E. Tetlock, \& E. G. Carmines (Eds.), Prejudice, politics, and the American dilemma (pp. 127-147). Stanford, CA: Stanford University Press.

Lepore, L., \& Brown, R. (1997). Category and stereotype activation: Is prejudice inevitable? Journal of Personality and Social Psychology, 72, 275-287.

Lerner, M. J. (1980). The belief in a just world: A fundamental delusion. New York: Plenum.

Levy, S. R., Stroessner, S. J., \& Dweck, C. S. (1998). Stereotype formation and endorsement: The role of implicit theories. Journal of Personality and Social Psychology, 76, 1421-1436.

Lopez, G. E., Gurin, P., \& Nagda, B. A. (1998). Education and understanding structural causes for group inequalities. Political Psychology, 19, 305-329.

Malle, B. F. (2004). How the mind explains behavior: Folk explanations, meaning, and social interaction. Cambridge, MA: MIT Press.

McConahay, J. B. (1986). Modern racism, ambivalence, and the modern racism scale. In J. F. Dovidio \& S. L. Gaertner (Eds.), Prejudice, discrimination, and racism. London: Academic Press.

Michela, J. L., \& Wood, J. V. (1986). Causal attributions in health and illness. In P. C. Kendall (Ed.), Advances in cognitivebehavioral research and therapy (Vol. 5, pp. 179-235). New York: Academic Press.

Monteith, M. J. (1993). Self-regulation of prejudiced responses: Implications for progress in prejudice-reduction efforts. Journal of Personality and Social Psychology, 65, 469-485.

Monteith, M. J., Ashburn-Nardo, L., Voils, C. I., \& Czopp, A. M. (2002). Putting the brakes on prejudice: On the development and operation of cues for control. Journal of Personality and Social Psychology, 83, 1029-1050.

Monteith, M. J., \& Voils, C. I. (1998). Proneness to prejudiced responses: Toward understanding the authenticity of self-reported discrepancies. Journal of Personality and Social Psychology, 75, 901-916.

Monteith, M. J., Devine, P. G., \& Zuwerink, J. R. (1993). Self-directed versus other-directed affect as a consequence of prejudice-related discrepancies. Journal of Personality $\mathcal{E}$ Social Psychology, 64, 198-210.

Moskowitz, G. B., Gollwitzer, P. M., Wasel, W., \& Schaal, B. (1999). Preconscious control of stereotype activation through chronic egalitarian goals. Journal of Personality and Social Psychology, $77,167-184$.

O’Laughlin, M. J., \& Malle, B. F. (2002). How people explain actions performed by groups and individuals. Journal of Personality and Social Psychology, 82, 33-48.

Oskamp, S. (Ed.). (2000). Reducing prejudice and discrimination. Mahwah, NJ: Lawrence Erlbaum.

Pettigrew, T. F. (1979). The ultimate attribution error: Extending Allport's congnitive analysis of prejudice. Personality and Social Psychology Bulletin, 5, 461-476.

Plant, E. A., \& Devine, P. G. (1998). Internal and external motivation to respond without prejudice. Journal of Personality and Social Psychology, 75, 811-832.

Reyna, C., Henry, P. J., Korfmacher, W., \& Tucker, A. (2005). Examining the principles in principled conservatism: The role of responsibility stereotypes as cues for deservingness in racial policy decisions. Journal of Personality and Social Psychology, 90, 109-128.

Ross, L. (1977). The intuitive psychologist and his shortcomings: Distortions in the attribution process. In L. Berkowitz (Ed.), Advances in experimental social psychology (Vol. 10, pp. 173-220). New York: Academic Press.

Ross, M., \& Miller, D. T. (2002). The justice motive in everyday life. Cambridge, UK: Cambridge University Press.

Sidanius, J., \& Pratto, F. (1999). Social dominance: An intergroup theory of social hierarchy and oppression. New York: Cambridge University Press.

Taylor, D. M., \& Jaggi, V. (1974). Ethnocentrism and causal attribution in a South Indian context. Journal of Cross-Cultural Psychology, 5, 162-171.

Weiner, B. (1986). An attributional theory of motivation and emotion. New York: SpringerVerlag.

Weiner, B. (2006). Social motivation, justice, and the moral emotions: An attributional approach. Mahwah, NJ: Erlbaum.

Yzerbyt, V. Y., \& Rocher, S. (2002). Subjective essentialism and the emergence of stereotypes. In C. McGarty, \& V. Yzerbyt (Eds.), Stereotypes as explanations: The formation of meaningful beliefs about social groups (pp. 38-66). New York: New York University Press.

Yzerbyt, V. Y., Rocher, S., \& Schadron, G. (1997). Stereotypes as explanations: A subjective essentialist view of group perception. In R. Spears, P. Oakes, N. Ellemers, \& 
S. A. Haslam (Eds.), The social psychology of stereotyping and group life (pp. 20-50). Cambridge, MA: Blackwell.

Paper received 1 December 2005; revised version accepted 1 July 2006.

\section{Appendix A}

\section{Social Explanations Questionnaire}

NOTES:

- 'EXT' indicates items composing the external explanations scale.

- 'INT' indicates items composing the internal explanations scale.

- There are twice as many internal as external items. The reason is that six of the internal items were intended to tap biological essentialism (Keller, 2005; items 5, 6, 11, 12, 17, 18) whereas the other six internal items were intended to tap internal explanations with no particular biological implication (items 3, 4, 9, 10, 15, 16). These two sets of items behave identically in all analyses and so were simply averaged together.

EXT (1) The history of slavery, segregation, and discrimination suffered by African Americans has surely contributed to any current economic and social problems they are facing.

EXT (2) Ultimately, any social or economic problems of the African American community are rooted in the profound mistreatment they have been subjected to in the United States.

INT (3) African Americans are generally less well-off than are White Americans because they do not try as hard as White Americans.

INT (4) African Americans simply have different attitudes and values from White Americans and thus they will never fit in and be successful in the United States.

INT (5) African Americans are genetically inferior to White Americans, and I think this plays a role in African Americans' relatively low social and economic status.

INT (6) African Americans and White Americans evolved biologically under different circumstances and this has resulted in different levels of intelligence and ambition.

EXT (7) When I think about the history of African Americans in the United States, it is easy to understand why some of them feel angry or resentful; I would feel the same way.

EXT (8) The reason that African Americans are sometimes less likely to go to college than are White Americans is that African American schools in many neighborhoods are underfunded and inadequate.

INT (9) African Americans have fewer job opportunities because they are so hard to get along with; they are often argumentative and have attitude problems.

INT (10) The values of individual responsibility and hard work seem incompatible with African American culture and this does not bode well for their future.

INT (11) Sometimes I think that the reason many African Americans have poor educational outcomes is that they are just not a very intelligent group.

INT (12) It should come as no surprise that African Americans and White Americans do not have the same outcomes socially and economically: they are different races and different races have different inborn abilities.

EXT (13) Poverty is like a trap that is very difficult to escape; this is an important reason why African Americans continue to linger behind White Americans economically.

EXT (14) I imagine that the constant barrage of stereotypes and prejudice in the US is disheartening and debilitating for African Americans.

INT (15) African Americans have developed a culture of disrespecting themselves and others; they need to change this in order to improve their status in the United States.

INT (16) As soon as African Americans manage to get their culture focused on more positive values such as hard work and self-discipline, their social position will improve.

INT (17) African Americans are just naturally prone to violence; people who point to poverty and discrimination as causes of this violence are only making excuses.

INT (18) Biologically speaking, African Americans appear to be inherently better suited for physical rather than intellectual pursuits. 


\section{Appendix B}

\section{Social Reactions Questionnaire}

Imagine that you are alone, walking down a dark, urban street in the middle of the night. You are entering a dangerous, high-crime area. As you make your way down the street, you spot a group of African American male teenagers hanging out on the street corner. How likely would you be to engage in some behavior to avoid or keep your distance from the group?

Check ONE:

I would definitely NOT try to avoid or keep my distance.

_I might try to avoid or keep my distance.

For participants who checked the lower box, the following items were presented:

Compunction items:

- I feel a little guilty at the thought that I would avoid them

- I am ashamed at the thought that I would discriminate

Emotional distress items:

- It makes me sad that I feel I need to avoid certain people

- I am not bothered by imagining myself doing this

- I am distressed
Justice item:

- I worry that I might be unfair in avoiding or keeping my distance

Rationality items:

- I think I'm only being sensible

- I think I'm just being rational

- I cannot give people the benefit of the doubt if there is potential danger

- It's not my fault that I need to avoid such teenagers

\section{Biographical notes}

MICHAEL J. GILL is an associate professor in the department of psychology at Lehigh University in Bethlehem, Pennsylvania. He is interested in how social orientations (e.g. compassionate vs. punitive) are shaped by the explanatory frameworks people use to understand others' behavior.

MICHAEL R. ANDREYCHIK is a doctoral student in the department of psychology at Lehigh University in Bethlehem, Pennsylvania. He is interested in stereotyping and prejudice, the bases of prosocial orientations, and the impact of belief systems on intergroup and interpersonal processes. 